\title{
TÁC ĐộNG CỦA ĐỔI MỚI CÔNG NGHỆ ĐẾN HIỆU QUẢ CỦA CÁC NGÂN HÀNG THƯƠNG MẬI VIẸTT NAM
}

\author{
NGUYẼ̃N THỊ MỸ PHƯợNG, ĐẶNG THỊ TRƯỜNG GIANG \\ Khoa Tài chính - Ngân hàng, Trường Đại học Công nghiệp thành phố Hồ Chí Minh \\ nguyenthimyphuong@iuh.edu.vn
}

Tóm tắt. Bài viết nhằm mục tiêu nghiên cứu tác động của đổi mới công nghệ đến hiệu quả của các ngân hàng thương mại (NHTM) Việt Nam trong giai đoạn 2008-2018. Để đo lường hiệu quả và chỉ số đổi mới công nghệ của các NHTM Việt Nam, phương pháp phân tích bao dữ liệu (Data Envelopment Analysis DEA) được áp dụng dựa trên hai biến đầu ra và ba biến đầu vào. Sau đó, nghiên cứu sử dụng mô hình hồi quy Tobit để xem xét tác động của đổi mới công nghệ đến hiệu quả của các NHTM Việt Nam. Kết quả nghiên cứu cho thấy: Chỉ số đồi mới công nghệ trung bình của các NHTM Việt Nam ở mức $0,976<1$, giảm 2,4\% toàn giai đoạn. Trong khi đó, hiệu quả chi phí trung bình của các NHTM Việt Nam ở mức 78,74\%, cho thấy các NHTM Việt Nam chưa sử dụng tối đa các nguồn lực đầu vào. Kết quả mô mình Tobit chỉ ra rằng chỉ số đổi mới công nghệ có tác động cùng chiều đến hiệu quả chi phí của các NHTM Việt Nam. Ngoài ra, nghiên cứu này cũng cho thấy khả năng sinh lời, tăng trưởng kinh tế, lãi suất có tác động cùng chiều, trong khi đó sở hữu nhà nước, nợ xấu và lạm phát tác động ngược chiều đến hiệu quả chi phí của các NHTM Việt Nam.

Từ khóa. DEA, đổi mới công nghệ, hiệu quả ngân hàng, TOBIT

\section{THE IMPACT OF TECHNOLOGICAL INNOVATION ON THE EFFICIENCY OF VIETNAMESE COMMERCIAL BANKS}

\begin{abstract}
The paper aims to study the impact of technological innovation on the efficiency of Vietnamese commercial banks for the period of 2008-2018. To measure banking efficiency score and the technological innovation index, Data Envelopment Analysis (DEA) is applied on two outputs and three input variables. Then, the research uses the Tobit regression model to examine the impact of technological innovation on the efficiencyof Vietnamese commercial banks. The results reveal that The average technological innovation index of Vietnamese commercial banks is at $0,976<1$, down $2.4 \%$ over the whole period. Meanwhile, the average cost efficiency of Vietnamese commercial banks is $78,74 \%$, showing that the Vietnamese commercial banks have not used the maximum input resources. Tobit model results indicate that technology innovation has a positive impact on the cost efficiency of Vietnamese commercial banks. In addition, this study also shows that profitability, economic growth, and interest rates are positively associated with the cost efficiency of Vietnam commercial banks, while state ownership, non-performing loan and inflation are negatively related to the cost effectiveness of Vietnam Commercial Bank.
\end{abstract}

Keywords. DEA, technological innovation, banking efficiency, TOBIT

\section{GIÓ́I THIỆU}

Trong bối cảnh của cuộc cách mạng công nghiệp 4.0 đã và đang diễn ra mạnh mẽ trên toàn cầu, đổi mới công nghệ được coi là một trong những công cụ quan trọng nhất có thể ảnh hưởng đến nền kinh tế nói chung cũng như ngành ngân hàng nói riêng tại hầu hết các quốc gia trên thế giới (Chaarani \& Abiad, 2018). DeYoung (2001) đã dự báo rằng tiến bộ công nghệ sẽ phá hủy các mô hình đang được sử dụng trong việc phát triển và cung cấp dịch vụ tại các NHTM và sẽ thay thế chúng bằng các mô hình mới thông qua việc thay đổi cách thức tổ chức ngân hàng, chiến lược kinh doanh, mối quan hệ của ngân hàng với khách hàng và tất cả các chức năng cụ thể dựa trên những yếu tố cốt lõi của kỹ thuật số gồm trí tuệ nhân tạo $(\mathrm{AI})$, vạn vật kết nối - Internet of Things (IoT) và dữ liệu lớn (Big Data).

Thực tế cho thấy, trong hơn hai thập kỷ qua, ngành ngân hàng trên thế giới đã bị ảnh hưởng đáng kể bởi sự tiến bộ nhanh chóng và vượt bậc của tốc độ đổi mới công nghệ (Ratten, 2008; Rishi \& Saxena, 2004). Đổi 
mới công nghệ đã làm cho việc thực hiện xử lý và truyền tải thông tin tại các NHTM được nhanh hơn, sản phẩm ngân hàng có thể dễ dàng được tiếp thị do mạng lưới quy mô được mở rộng hơn dựa trên một tập hợp các kết nối khu vực và toàn cầu, khả năng tiếp cận và nhận thức của khách hàng được nâng cao hơn, theo đó làm gia tăng đáng kể hiệu quả hoạt động ngân hàng (Campanella \& cộng sự, 2017).

Tại Việt Nam, đa số các NHTM đều khẳng định rằng đổi mới công nghệ có ý nghĩa quan trọng sống còn trong hoạt động kinh doanh và $96 \%$ ngân hàng đã và đang xây dựng chiến lược phát triển dựa trên các công nghệ 4.0 (NHNN, 2019). Mặc dù thực tế là các NHTM Việt Nam bị ảnh hưởng nhiều bởi sự đổi mới công nghệ trong bối cảnh cuộc cách mạng công nghiệp 4.0 đang diễn ra mạnh mẽ, nhưng tính đến nay, chưa có nghiên cứu thực nghiệm nào đi sâu nghiên cứu về tác động của của đổi mới công nghệ đến hiệu quả của các NHTM Việt Nam. Hầu hết các nghiên cứu trong nước như Vinh (2012), Trúc \& Danh (2012), Sáng (2013), Hoàng \& Huân (2016), Thương (2017) đều tập trung nghiên cứu về các yếu tố tác động đến hiệu quả ngân hàng, tuy nhiên tất cả các nghiên cứu này đều chưa tính đến tác động của đổi mới công nghệ đến hiệu quả của các NHTM Việt Nam. Do vậy, xuất phát từ bối cảnh khoa học và thực tiễn tại Việt Nam, nghiên cứu này được thực hiện với kỳ vọng mang lại đóng góp mới về phương pháp tiếp cận đo lường chỉ sổ đổi mới công nghệ và bổ sung vào khoảng trống nghiên cứu thông qua cung cấp bằng chứng thực nghiệm về tác động của đổi mới công nghệ đến hiệu quả của các NHTM Việt Nam.

\section{CƠ SỞ LÝ THUYÊTT}

\section{1 Đổi mới công nghệ}

Drucker (2013) cho rằng đổi mới là quá trình liên quan đến sự trang bị các khả năng mới, được cải thiện hoặc tiện ích gia tăng. Điều này có thể được thực hiện thông qua các sản phẩm, quy trình, dịch vụ, công nghệ hoặc ý tưởng hiệu quả mới có sẵn cho thị trường, chính phủ và xã hội (BCG, 2009). Trong khi đó, công nghệ là kiến thức và ứng dụng của các sản phẩm và quy trình, phương pháp, công cụ và hệ thống tạo ra hoặc sản xuất hàng hóa và dịch vụ (Khalil, 2000). Do đó, đổi mới công nghệ được tạo thành từ đổi mới hệ thống, đổi mới quy trình, đổi mới đội ngũ lao động và đổi mới trang thiết bị làm việc trong một tổ chức (Oke \& Goffin, 2011). Theo lý thuyết khuyếch tán đổi mới của Rogers (1962), trong ngành ngân hàng, đổi mới công nghệ có một năng lực đặc biệt làm gia tăng lợi thế cạnh tranh của ngân hàng trên cơ sở cho phép các ngân hàng tung ra các sản phẩm trong nền tảng ngân hàng của họ nhờ các hệ thống ngân hàng lõi vượt trội có nguồn gốc từ các công ty chuyên phát triển, sản xuất, kinh doanh bản quyền phần mềm và hỗ trợ trên diện rộng các sản phẩm và dịch vụ liên quan đến máy tính như IBM, Mysis và Microsoft (Langley \& cộng sự, 2011).

\subsection{Hiệu quả ngân hàng}

\subsubsection{Khái niệm hiệu quả ngân hàng}

Khái niệm về hiệu quả của ngân hàng vẫn còn được tranh cãi giữa các nhà nghiên cứu. Để xác định những gì tạo nên hiệu quả của các ngân hàng, trước tiên người ta nên quyết định về bản chất của phương pháp tiếp cận ngân hàng. Có hai cách tiếp cận chính được sử dụng rộng rãi trong các tài liệu lý thuyết ngân hàng, đó là phương pháp sản xuất và phương pháp trung gian (Sealey \& Lindley, 1977). Cách tiếp cận sản xuất giả định rằng ngân hàng đóng vai trò là nhà cung cấp dịch vụ cho chủ tài khoản; nghĩa là, họ nên thực hiện các giao dịch trên tài khoản tiền gửi và xử lý các khoản vay. Cách tiếp cận trung gian theo quan điểm là các ngân hàng về cơ bản đóng vai trò là trung gian tài chính với vai trò chính là lây tiền từ người tiết kiệm để đổi lấy các khoản nợ của họ, và đến lượt các ngân hàng sẽ cung cấp các khoản vay cho người khác để kiếm lợi nhuận (Chu \& Lim, 1998). Berger \& Humphrey (1992) lập luận rằng cả hai cách tiếp cận này đều không hoàn hảo vì họ không thể nắm bắt hoàn toàn vai trò kép của ngân hàng với tư cách là nhà cung cấp dịch vụ/giao dịch và cũng là trung gian tài chính. Họ chỉ ra rằng phương pháp sản xuất có thể tốt hơn đề đánh giá hiệu quả của các chi nhánh ngân hàng và phương pháp trung gian có thể phù hợp hơn để đánh giá toàn bộ các ngân hàng. Kablan (2009) cho rằng hiệu quả ngân hàng là thước đo mức độ quản lý phù hợp với công nghệ, quản lý nguồn nhân lực và các nguồn lực khác để tạo ra một mức sản lượng nhất định. Trong khi đó, theo Farrell (1957), hiệu quả ngân hàng có thể được chia thành hai thành phần, gồm hiệu quả kỹ thuật (TE) và hiệu quả phân bổ (AE).

Hiệu quả chi phí $(\mathrm{CE})=$ Hiệu quả kỹ thuật $(\mathrm{TE})$ x Hiệu quả phân bổ $(\mathrm{AE})=(\mathrm{OQ} / \mathrm{OP}) \mathrm{x}(\mathrm{OR} / \mathrm{OQ})$

\subsection{2 Đo luờng hiệu quả ngân hàng}


THƯƠNG MẠI VIẸTT NAM

Theo Wozniewska (2008), hiệu quả ngân hàng thường được đo lường thông qua phương pháp phân tích hiệu quả biên. Phương pháp này tính toán chỉ số hiệu quả tương đối dựa trên việc so sánh khoảng cách của các đơn vị ra quyết định (Decision Making Unit - DMU) hay ngân hàng với một đơn vị thực hiện hoạt động tốt nhất trên biên. Công cụ này cho phép tính được chỉ số hiệu quả chung của từng ngân hàng dựa trên hoạt động của chúng và cho phép xếp hạng hiệu quả của các ngân hàng. Phương pháp phân tích hiệu quả biên có thể được chia thành 2 nhóm gồm: (i) Phương pháp tham số - Phân tích biên ngẫu nhiên (The Stochastic Frontier Approach - SFA), được giới thiệu bởi Aigner \& cộng sự (1977) và (ii) Phương pháp phi tham số Phân tích bao dữ liệu (Data envelopment approach - DEA) được đề xuất bởi Charnes \& cộng sự (1978), sau đó là Banker \& cộng sự (1984). Mặc dù cả hai phương pháp này đều có ưu và nhược điểm trong đo lường hiệu quả, nhưng theo thời gian, các nhà nghiên cứu đã ủng hộ rằng DEA là một kỹ thuật vượt trội hơn so với SFA trong tính toán điểm hiệu quả tổng thể trên các ngành công nghiệp khác nhau (Chandra \& cộng sự, 1998; Kumar \& Arora, 2011; Kumar \& Gulati, 2009; Kumar, 2011).

\section{3 Đổi mới công nghệ và hiệu quả ngân hàng}

Hobe \& Alas (2016) chỉ ra rằng đổi mới công nghệ là một trong những động lực sinh lời chính của các ngân hàng và trong thế kỷ 21 , nó ngày càng trở nên quyết định trong hiệu quả và khả năng cạnh tranh của ngân hàng. Beccalli (2007) đã tiết lộ rằng việc đầu tư vào công nghệ thông tin và đổi mới có tác động đáng kể đến hiệu quả của ngành ngân hàng. Romdhane (2013) cho thấy rằng các khoản đầu tư vào internet và ngân hàng trên di động có thể làm tăng hiệu suất ngân hàng. Abaenewe \& cộng sự (2013) tìm thấy bằng chứng chỉ ra tác động tích cực của máy rút tiền tự động (ATM) và máy POS đến hiệu suất và lợi thế cạnh tranh trong lĩnh vực ngân hàng. Ngugi \& Karina (2013) đã nghiên cứu ảnh hưởng của các chiến lược đổi mới công nghệ đến hiệu quả các NHTM ở Kenya, họ thấy rằng các chiến lược như tái định vị sản phẩm, thay thế sản phẩm và chiến lược đổi mới quy trình như tuân thủ các quy định và giảm chi phí có tác động làm tăng hiệu quả ngân hàng. Họ kết luận rằng việc áp dụng các chiến lược đổi mới công nghệ có tác động cùng chiều đến hiệu quả ngân hàng. Trong khi đó, Yang \& Huang (2009) khẳng định rằng việc áp dụng công nghệ tài chính sẽ nâng cao hiệu quả và năng lực cạnh tranh tổng thể của ngành ngân hàng tại Đài Loan.

Từ những bằng chứng thực nghiệm nêu trên, cho thấy việc đổi mới công nghệ có tác động cùng chiều đến hiệu quả của các NHTM, theo đó đổi mới công nghệ là một trong những yếu tố quan trọng trong việc nâng cao hiệu quả và lợi thế cạnh tranh trong lĩnh vực ngân hàng.

\section{PHƯƠNG PHÁP NGHIÊN CÚU VÀ DŨ̉ LIẸU}

Bài viết sử dụng phương pháp nghiên cứu định lượng nhằm mục tiêu xác định mức độ tác động của đổi mới công nghệ đến hiệu quả của 22 NHTMVN, gồm ABB, ACB, BIDV, CTG, EIB, HDB, MBB, MSB, NAB, OCB, PGB, SCB, SEA, SGB, SHB, STB, TCB, TPB, VAB, VCB, VIB, VPB trong giai đoạn 20092018. Nghiên cứu tiến hành theo hai giai đoạn: (i) Đo lường hiệu quả và chỉ số đổi mới công nghệ của 22 NHTM Việt Nam trong giai đoạn 2008-2018 theo cách tiếp cận phân tích hiệu quả biên phi tham số bao dữ liệu DEA; (ii) Sử dụng mô hình hồi quy Tobit để xem xét tác động của đổi mới công nghệ đến hiệu quả của các NHTM Việt Nam.

\section{1 Đo lường hiệu quả và chỉ số đổi mới công nghệ của các ngân hàng thương mại Việt Nam}

\subsection{1 Đo lường hiệu quả của các ngân hàng thưong mại Việt Nam}

Để đo lường hiệu quả của 22 NHTM Việt Nam trong giai đoạn 2008-2018, tác giả sử dụng phương pháp DEA do kỹ thuật vượt trội của phương pháp này so với phương pháp tham số SFA (Chandra \& cộng sự, 1998; Kumar \& Arora, 2011; Kumar \& Gulati, 2009; Kumar, 2011). Theo Charnes \& cộng sự (1978), DEA là mô hình lập trình toán học được áp dụng cho dữ liệu quan sát cung cấp một cách mới để có được ước tính thực nghiệm về các mối quan hệ như các hàm sản xuất hoặc bề mặt khả năng sản xuất hiệu quả, được xem là nền tảng của kinh tế học hiện đại. Cách tiếp cận này cung cấp một giá trị hiệu quả số được xác định một cách khách quan bằng cách sử dụng nhiều đầu vào và đầu ra. DEA bắt đầu với một công thức lập trình phân đoạn tương đối đơn giản.

Giả sử rằng có $n \mathrm{DMU}$ hay ngân hàng được đánh giá. Mỗi loại tiêu thụ số lượng đầu vào $i$ khác nhau và tạo

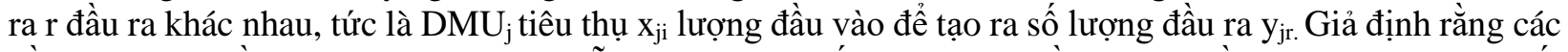
đầu vào $\mathrm{x}_{\mathrm{ji}}$ và đầu ra $\mathrm{y}_{\mathrm{jr}}$ không âm và mỗi DMU có ít nhất một giá trị đầu vào và đầu ra dương. Năng suất của DMU có thể được viết là: 


$$
h_{j}=\frac{\sum_{r=1}^{s} u_{r} y_{r j}}{\sum_{i=1}^{m} v_{i} x_{i j}}
$$

Trong công thức này, $\mathrm{u}$ và $\mathrm{v}$ là các trọng số được gán cho từng đầu vào và đầu ra. Bằng cách sử dụng các kỹ thuật lập trình toán học, DEA gán tối ưu các trọng số theo các ràng buộc sau: Các trọng số cho mỗi DMU được gán theo ràng buộc rằng không có $\mathrm{DMU}$ nào khác có hiệu suất lớn hơn 1 nếu nó sử dụng cùng các trọng số, ngụ ý rằng DMU hoàn toàn hiệu quả sẽ có giá trị là 1 .

Các trọng số dẫn xuất, u và v không âm. Hàm mục tiêu của $\mathrm{DMU}_{\mathrm{k}}$ là tỷ lệ của tổng đầu ra có trọng số chia cho tổng đầu vào có trọng số và có thể được trình bày theo công thức cơ bản sau:

$$
\begin{gathered}
\max h_{\mathrm{o}}(u, v)=\frac{\sum_{r=1}^{s} u_{r} y_{r \mathrm{O}}}{\sum_{i=1}^{m} v_{i} x_{i \mathrm{O}}} \\
\text { subjectto } h_{j}=\frac{\sum_{r=1}^{s} u_{r} y_{r j}}{\sum_{i=1}^{m} v_{i} x_{i j}} \leq 1, \quad j=1 \ldots n \\
u_{r} \geq 0, r=1,2, \ldots ., s, \\
v_{i} \geq 0, i=1,2, \ldots, m,
\end{gathered}
$$

\subsection{2 Đo lường chỉ số đổi mới công nghệ của các ngân hàng thuơng mại Việt Nam}

Dựa trên các nghiên cứu của Liu \& Pan (2007), Yuan \& cộng sự (2007), Wuqin \& Shunlin (2008), Yun (2011), Cruz-Cázares \& cộng sự (2013), nhóm tác giả sử dụng phương pháp DEA theo cách tiếp cận chỉ số Malmquist để phân rã thành chỉ số đổi mới công nghệ (phản ánh sự thay đổi tiến bộ công nghệ) để đo lường chỉ số đổi mới công nghệ của 22 NHTM Việt Nam.

Theo Färe \& cộng sự (1994), chỉ số Malmquist được xác định như sau:

$$
M_{0}\left(x^{t+1}, y^{t+1}, x^{t}, y^{t}\right)=\left[\left(\frac{D_{0}^{t}\left(x^{t+1}, y^{t+1}\right)}{D_{0}^{t}\left(x^{t}, y^{t}\right)}\right)\left(\frac{D_{0}^{t+1}\left(x^{t+1}, y^{t+1}\right)}{D_{0}^{t+1}\left(x^{t}, y^{t}\right)}\right)\right]^{1 / 2}
$$

Trong đó, giả thiết rằng tương ứng với mỗi thời kỳ $\mathrm{t}=1, \ldots, \mathrm{T}$ có công nghệ sản xuất $\mathrm{H}^{\mathrm{T}}$ biểu thị cách kết hợp tất cả các đầu ra $\mathrm{y}^{\mathrm{t}}$ có thể được sản xuất bằng cách sử dụng đầu vào $\mathrm{x}^{\mathrm{t}}$. Giả định rằng $\mathrm{H}^{\mathrm{t}}$ thỏa mãn một số tiêu chuẩn nhất định để xác định hàm khoảng cách đầu ra như sau:

$$
D_{0}^{t}\left(x^{t}, y^{t}\right)=\inf \left\{\lambda:\left(x^{t}, y^{t} / \lambda\right) \in H^{t}\right\}
$$

Từ đó, phân rã (4) thành các thành phần như sau:

$$
M_{0}\left(x^{t+1}, y^{t+1}, x^{t}, y^{t}\right)=\left(\frac{D_{0}^{t+1}\left(x^{t+1}, y^{t+1}\right)}{D_{0}^{t}\left(x^{t}, y^{t}\right)}\right)\left[\left(\frac{D_{0}^{t}\left(x^{t+1}, y^{t+1}\right)}{D_{0}^{t+1}\left(x^{t+1}, y^{t+1}\right)}\right)\left(\frac{D_{0}^{t+1}\left(x^{t}, y^{t}\right)}{D_{0}^{t+1}\left(x^{t}, y^{t}\right)}\right)\right]^{1 / 2}
$$

Trong đó, thành phần $\left[\left(\frac{D_{0}^{t}\left(x^{t+1}, y^{t+1}\right)}{D_{0}^{t+1}\left(x^{t+1}, y^{t+1}\right)}\right)\left(\frac{D_{0}^{t+1}\left(x^{t}, y^{t}\right)}{D_{0}^{t+1}\left(x^{t}, y^{t}\right)}\right)\right]$ thể hiện sự đổi mới công nghệ biên giữa hai thời

kỳ $\mathrm{t}$ và $\mathrm{t}+1$.

\subsubsection{Dũ liệu các biến trong mô hình DEA}

Để lựa chọn các biến trong phân tích DEA nhằm đo lường hiệu quả và chỉ số đổi mới công nghệ của các NHTM Việt Nam, nghiên cứu dựa trên cách tiếp cận trung gian theo quan điểm của Berger \& Humphrey (1992). Theo cách tiếp cận này, các biến đầu vào (Input), đầu ra (Output) và biến giá các đầu vào (Input Price) của các NHTM Việt Nam được trình bày tại Bảng $1 \&$ kết quả thống kê mô tả các biến này được 
trình bày tại Bảng 2. Nguồn dữ liệu các biến được thu thập từ báo cáo tài chính (BCTC) của 22 NHTM Việt Nam trong giai đoạn 2008-2018.

Bảng 1: Các biến đầu vào, đầu ra và biến giá của các đầu vào trong phân tích DEA

\begin{tabular}{|l|c|l|c|}
\hline \multicolumn{1}{|c|}{ Biến } & Ký hiệu & \multicolumn{1}{|c|}{ Cách tính } & Đơn vị tính \\
\hline OUTPUT & & & \\
\hline Thu lãi và các khoản tương đương & Y1 & Thu lãi và các khoản tương đương & Triệu đồng \\
\hline Thu ngoài lãi và các khoản tương đương & Y2 & Thu ngoài lãi và các khoản tương đương & Triệu đồng \\
\hline INPUT & & & \\
\hline Chi phí lao động & $\mathrm{X} 1$ & Chi phí cho nhân viên & Triệu đồng \\
\hline Tổng tài sản cố định ròng & $\mathrm{X} 2$ & Tổng tài sản cố định ròng & Triệu đồng \\
\hline Tổng vốn huy động từ khách hàng & $\mathrm{X} 3$ & Tổng vốn huy động từ khách hàng & Triệu đồng \\
\hline INPUT PRICE & & & Chi cho nhân viên/Tổng số nhân viên \\
\hline Giá của lao động & $\mathrm{P} 1$ & Triệu \\
\hline Giá của tài sản & $\mathrm{P} 2$ & Chi về tài sản/Tổng tài sản cố định ròng & Lần \\
\hline Giá của vốn huy động & $\mathrm{P} 3$ & $\begin{array}{l}\text { Chi trả lãi và các khoản tương đương/Tổng } \\
\text { vôn huy động từ khách hàng }\end{array}$ & Lần \\
\hline
\end{tabular}

Nguồn: Tổng hợp và đề xuất của nhóm tác giả

Bảng 2: Kết quả thống kê mô tả các biến trong phân tích DEA

\begin{tabular}{|c|c|r|r|r|r|}
\hline Biến & Số quan sát & \multicolumn{1}{c|}{ Trung bình } & \multicolumn{1}{c|}{ Độ lệch chuẩn } & \multicolumn{1}{c|}{ Giá trị nhỏ nhất } & \multicolumn{1}{c|}{ Giá trị lớn nhất } \\
\hline Y1 & 242 & 12.400 .000 & 14.700 .000 & 198.427 & 90.100 .000 \\
\hline Y2 & 242 & 1.463 .048 & 1.818 .389 & 2.219 & 8.418 .656 \\
\hline X1 & 242 & 1.472 .183 & 2.446 .785 & 19.571 & 22.200 .000 \\
\hline X2 & 242 & $983.918,6$ & 1.327 .780 & 41.536 & 6.422 .388 \\
\hline X3 & 242 & 120.000 .000 & 166.000 .000 & 1.171 .843 & 990.000 .000 \\
\hline P1 & 242 & 174,329 & 78,896 & 53,45 & 446,7 \\
\hline P2 & 242 & 6,192 & 5,483 & 0,04 & 34,12 \\
\hline P3 & 242 & 0,088 & 0,049 & 0 & 0,29 \\
\hline
\end{tabular}

Nguồn: Tính toán của nhóm tác giả

3.2 Mô hình nghiên cứu tác động của đổi mới công nghệ đến hiệu quả của các ngân hàng thương mại Việt Nam

Để xác định tác động của đổi mới công nghệ đến hiệu quả của các NHTMVN, tác giả sử dụng mô hình Tobit được phát triển bởi Tobin (1958) với biến phụ thuộc là hiệu quả ngân hàng. Cách tiếp cận này được sử dụng trong các nghiên cứu của Havrylchyk (2006), Ariff \& Can (2008), Sari \& Saraswati (2017), Batir \& cộng sự (2017) để phân tích các yếu tố tác động đến hiệu quả ngân hàng. Theo Gujarati (2003), mô hình Tobit được sử dụng trong phân tích hồi quy cho trường hợp biến phụ thuộc bị kiểm duyệt.

Mô hình hồi quy Tobit có dạng như sau:

$$
\begin{aligned}
\mathrm{Y}^{*}= & \beta_{0}+\sum_{i=1}^{n} \beta_{i} X_{i}+\sum_{j=1}^{n} \beta_{j} D_{j}+\delta_{i}, \delta_{i} \sim \mathrm{N}\left(0, \sigma^{2}\right) \text { với } 0<\mathrm{Y}^{*}<1 \\
& \begin{cases}Y=Y^{*}, & Y^{*}>0 \\
Y=0, & Y^{*}<0\end{cases}
\end{aligned}
$$


Trong đó:

$\mathrm{Y}_{\mathrm{i}}^{*}$ là các biến phụ thuộc phản ánh hiệu quả chi phí của ngân hàng được ước lượng bằng phương pháp hợp lý cực đại

$\mathrm{Y}_{\mathrm{i}}$ là biến phụ thuộc phản ánh hiệu quả chi phí của ngân hàng được đo lường từ phương pháp DEA

$\mathrm{X}_{\mathrm{i}}$ là các biến độc lập; $\mathrm{D}_{\mathrm{j}}$ là các biến kiểm soát; $\beta_{\mathrm{i}}, \beta_{\mathrm{j}}$ là các tham số được ước lượng; $\delta_{\mathrm{i}}$ là sai số theo phân phối chuẩn

Các biến phụ thuộc, độc lập và các biến kiểm soát của mô hình Tobit trình bày tại Bảng 3 , được nhóm tác giả đề xuất dựa trên các nghiên cứu trước của Mercan \& cộng sự (2003), Williams \& Nguyen (2005), Rezitis (2007), Hassan \&Sanchez (2007), Havrylchyk (2006), Ariff \& Can (2008), Sufian \& Noor (2009), Vu \& Nahm (2013), GarzaGarcía (2012), Said \& cộng sự (2013), Adjei-Frimpong \& cộng sự (2014), Batir \& cộng sự (2017), Sari \& Saraswati (2017); bối cảnh thực tiễn và nguồn số liệu sẵn có của Việt Nam được thu thập từ Thống kê Tài chính Quốc tế (International Financial Statistics - IFS) của Quỹ Tiền tệ Quốc tế, Tổng Cục Thống kê Việt Nam (General Statistics Office of Vietnam - GSO) và BCTC của 22 NHTM Việt Nam.

Bảng 3: Các biến sử dụng trong mô hình Tobit

\begin{tabular}{|c|c|c|c|c|c|}
\hline Biến & Ký hiệu & Cách đo lường & $\begin{array}{c}\text { Kỳ } \\
\text { vọng }\end{array}$ & $\begin{array}{l}\text { Nguồn } \\
\text { dữ liệu }\end{array}$ & Nghiên cứu tham khảo \\
\hline \multicolumn{6}{|l|}{ Biến phụ thuộc } \\
\hline Hiệu quả chi phí & $\mathrm{CE}$ & Ước lượng từ DEA & & BCTC & $\begin{array}{l}\text { Havrylchyk (2006); Ariff } \\
\text { \& Can (2008); Sari \& } \\
\text { Saraswati (2017); Batir } \\
\text { \& cộng sự (2017); }\end{array}$ \\
\hline \multicolumn{6}{|l|}{ Biến độc lập } \\
\hline Đổi mới công nghệ & TECH & $\begin{array}{l}\text { Ước lượng từ DEA dựa } \\
\text { trên chỉ số Malmquist }\end{array}$ & + & BCTC & $\begin{array}{l}\text { Liu \& Pan (2007), Yuan } \\
\text { \& cộng sự (2007), Wuqin } \\
\text { \& Shunlin (2008), Yun } \\
\text { (2011), Cruz-Cázares \& } \\
\text { cộng sự (2013); }\end{array}$ \\
\hline \multicolumn{6}{|l|}{ Biến kiểm soát } \\
\hline Khả năng sinh lời & ROA & $\begin{array}{l}\text { Lợi } \quad \text { nhuận } \\
\text { thuê/Tồng tài sản }\end{array}$ & + & BCTC & $\begin{array}{l}\text { Sari \& Saraswati (2017); } \\
\text { Adjei-Frimpong \& cộng } \\
\text { sự (2014); Hassan \& } \\
\text { Sanchez (2007); Sufian } \\
\text { \& Noor (2009); }\end{array}$ \\
\hline Nợ xấu & NPL & $\begin{array}{l}\text { Nợ xấu/tổng dư nợ tín } \\
\text { dụng }\end{array}$ & - & BCTC & $\begin{array}{l}\text { Havrylchyk (2006); Vu } \\
\text { \& Nahm (2013); Sufian } \\
\text { \& Noor (2009); }\end{array}$ \\
\hline Qui mô ngân hàng & SIZE & $\begin{array}{l}\text { Logarithm tự nhiên của } \\
\text { Tống tài sản }\end{array}$ & + & BCTC & $\begin{array}{l}\text { Vu \& Nahm (2013); } \\
\text { Mercan \& cộng sự } \\
\text { (2003); Williams \& } \\
\text { Nguyen (2005); Rezitis } \\
\text { (2007), Sufian \& Noor } \\
\text { (2009), Said \& cộng sự } \\
\text { (2013); }\end{array}$ \\
\hline $\begin{array}{l}\text { Vốn chủ sở hữu/Tổng tài } \\
\text { sản }\end{array}$ & EA & $\begin{array}{l}\text { Vốn chủ sở hữu/Tổng } \\
\text { tài sản }\end{array}$ & + & BCTC & $\begin{array}{l}\text { Said \& cộng sự (2013); } \\
\text { Vu \& Nahm (2013); }\end{array}$ \\
\hline Sở hữu nhà nước & SO & $\begin{array}{l}\text { Biến giả, nhận giá trị } 1 \\
\text { nếu là ngân hàng có vốn } \\
\text { đầu tư Nhà nước và } 0 \\
\text { nếu ngược lại }\end{array}$ & - & BCTC & Vu \& Nahm (2013); \\
\hline
\end{tabular}


THƯONG MẠI VIỆT NAM

\begin{tabular}{|c|c|c|c|c|c|}
\hline Biến & Ký hiệu & Cách đo lường & $\begin{array}{c}\text { Kỳ } \\
\text { vọng }\end{array}$ & $\begin{array}{l}\text { Nguồn } \\
\text { dữ liệu }\end{array}$ & Nghiên cứu tham khảo \\
\hline Sở hữu nước ngoài & FO & $\begin{array}{l}\text { Biến giả, nhận giá trị } 1 \\
\text { nếu là ngân hàng có vốn } \\
\text { đầu tư nước ngoài và } 0 \\
\text { nếu ngược lại }\end{array}$ & + & BCTC & Havrylchyk (2006); \\
\hline Tăng trưởng kinh tế & GDP & Tăng trưởng GDP & + & GSO & $\begin{array}{lll}\text { Vu \& Nahm } & \text { (2013); } \\
\text { GarzaGarcía } & \text { (2012); } \\
\text { Hassan \& } & \text { Sanchez } \\
(2007) ; & & \end{array}$ \\
\hline Lạm phát & INF & Tăng trưởng CPI & - & IFS & $\begin{array}{lcr}\text { Vu \& } & \text { Nahm } & \text { (2013); } \\
\text { Hassan } & \& & \text { Sanchez } \\
\text { (2007); } & & \end{array}$ \\
\hline Lãi suất & RATE & $\begin{array}{l}\text { Chênh lệch giữa lãi suất } \\
\text { cho vay và lãi suất tiền } \\
\text { gửi }\end{array}$ & + & IFS & $\begin{array}{lcr}V u \quad \& & \text { Nahm } & (2013) \\
\text { Hassan } & \& & \text { Sanchez } \\
\text { (2007); } & & \end{array}$ \\
\hline
\end{tabular}

Nguồn: Tổng hợp và đề xuất của nhóm tác giả

Theo Bảng 2, mô hình hồi quy Tobit xác định tác động của đổi mới công nghệ đến hiệu quả của các NHTMVN được viết lại cụ thể theo phương trình sau:

$\mathrm{CE}=\beta_{0}+\beta_{1} \mathrm{TECH}+\beta_{2} \mathrm{ROA}+\beta_{3} \mathrm{NPL}+\beta_{4} \mathrm{SIZE}+\beta_{5} \mathrm{EA}+\beta_{6} \mathrm{GDP}+\beta_{7} \mathrm{INF}+\beta_{8} \mathrm{RATE}+\delta_{\mathrm{i}}$

Từ đó, nghiên cứu này đặt ra giả thuyết $\mathrm{H} 1$ : "Đổi mới công nghệ có tác động cùng chiều đến hiệu quả của các NHTM Việt Nam".

\section{Kết quả nghiên cứu và thảo luận}

\subsection{Hiệu quả của các ngân hàng thương mại Việt Nam trong giai đoạn 2008-2018}

Kết quả ướng lượng DEA tại Bảng 4 cho thấy, hiệu quả chi phí (hiệu quả kinh tế toàn bộ) trung bình của các NHTM Việt Nam trong giai đoạn 2008-2018 ở mức 78,74\%, trong đó hiệu quả kỹ thuật trung bình ở mức $92,18 \%$ và hiệu quả phân bổ trung bình ở mức $84,92 \%$, cho thấy trong thời gian qua để tạo ra cùng một mức sản lượng đầu ra như nhau thì các NHTM Việt Nam đã chưa sử dụng tối đa các nguồn lực đầu vào của mình và còn lãng phí các đầu vào ở mức trung bình $27 \%$. Các ngân hàng có hiệu quả chi phí trung bình thấp hơn mức trung bình chung của cả mẫu nghiên cứu gồm có $\mathrm{ABB}(55,92 \%)$, EIB $(59,33 \%)$, SHB $(61,58 \%), \operatorname{HDB}(61,99 \%), \operatorname{MBB}(65,46 \%), \operatorname{ACB}(69,74 \%), \operatorname{VIB}(71,2 \%), \operatorname{SEA}(72,96 \%), \operatorname{OCB}(77,11 \%)$, NAB $(78,08 \%)$. Các NHTM Việt Nam có hiệu quả chi phí trung bình cao hơn mức trung bình chung của cả mẫu nghiên cứu, gồm có BIDV $(98,79 \%)$, VCB $(96,57 \%)$, TCB $(94,23 \%)$, SCB $(90,63 \%)$, STB $(89,55 \%)$, CTG $(89,54 \%)$, VAB $(87,28 \%)$, SGB $(84,87 \%)$, MSB $(83,61 \%)$, VPB (82,73\%), PGB $(80,90 \%)$, TPB (80,29\%). Xét trong toàn giai đoạn nghiên cứu, năm 2008, 2011, 2012, 2015 - 2018 là các năm các NHTM Việt Nam có hiệu quả chi phí trung bình thấp hơn mức trung bình chung của toàn giai đoạn, trong đó năm 2012 là năm các NHTM có hiệu quả chi phí trung bình thấp nhất ở mức $68,8 \%$ và nhìn chung hiệu quả của các NHTM Việt Nam đều giảm dần theo thời gian (Hình 2).

Bảng 4: Hiệu quả kỹ thuật, hiệu quả phân bổ và hiệu quả chi phí trung bình của các NHTM Việt Nam trong giai đoạn 2008-2018

\begin{tabular}{|c|c|c|c|}
\hline Ngân hàng & $\begin{array}{c}\text { Hiệu quả kỹ thuật } \\
\text { (TE) }\end{array}$ & $\begin{array}{c}\text { Hiệu quả phân bổ } \\
\text { (AE) }\end{array}$ & $\begin{array}{c}\text { Hiệu quả chi phí } \\
\text { (CE) }\end{array}$ \\
\hline ABB & 0,7361 & 0,7605 & 0,5592 \\
\hline ACB & 0,8642 & 0,7924 & 0,6974 \\
\hline BIDV & 0,9987 & 0,9891 & 0,9879 \\
\hline CTG & 0,9552 & 0,9319 & 0,8954 \\
\hline EIB & 0,8001 & 0,7548 & 0,5933 \\
\hline HDB & 0,8368 & 0,7382 & 0,6199 \\
\hline MBB & 0,8483 & 0,7900 & 0,6546 \\
\hline
\end{tabular}



THƯONG MẠI VIẸT NAM

\begin{tabular}{|c|c|c|c|}
\hline Ngân hàng & $\begin{array}{c}\text { Hiệu quả kỹ thuật } \\
\text { (TE) }\end{array}$ & $\begin{array}{c}\text { Hiệu quả phân bổ } \\
\text { (AE) }\end{array}$ & $\begin{array}{c}\text { Hiệu quả chi phí } \\
\text { (CE) }\end{array}$ \\
\hline MSB & 0,9765 & 0,8528 & 0,8361 \\
\hline NAB & 0,9617 & 0,8045 & 0,7808 \\
\hline OCB & 0,9328 & 0,8187 & 0,7711 \\
\hline PGB & 0,9453 & 0,8501 & 0,8090 \\
\hline SCB & 0,9212 & 0,9805 & 0,9063 \\
\hline SEA & 0,8979 & 0,8101 & 0,7296 \\
\hline SGB & 0,9420 & 0,8958 & 0,8487 \\
\hline SHB & 0,9126 & 0,6851 & 0,6158 \\
\hline STB & 0,9552 & 0,9319 & 0,8955 \\
\hline TCB & 0,9946 & 0,9456 & 0,9423 \\
\hline TPB & 1,0000 & 0,8029 & 0,8029 \\
\hline VAB & 0,9613 & 0,9101 & 0,8728 \\
\hline VCB & 0,9657 & 0,9994 & 0,9657 \\
\hline VIB & 0,9272 & 0,7671 & 0,7120 \\
\hline VPB & 0,9475 & 0,8708 & 0,8273 \\
\hline Trung bình & $\mathbf{0 , 9 2 1 8}$ & $\mathbf{0 , 8 4 9 2}$ & $\mathbf{0 , 7 8 7 4}$ \\
\hline
\end{tabular}

Nguồn: Tính toán của nhóm tác giả

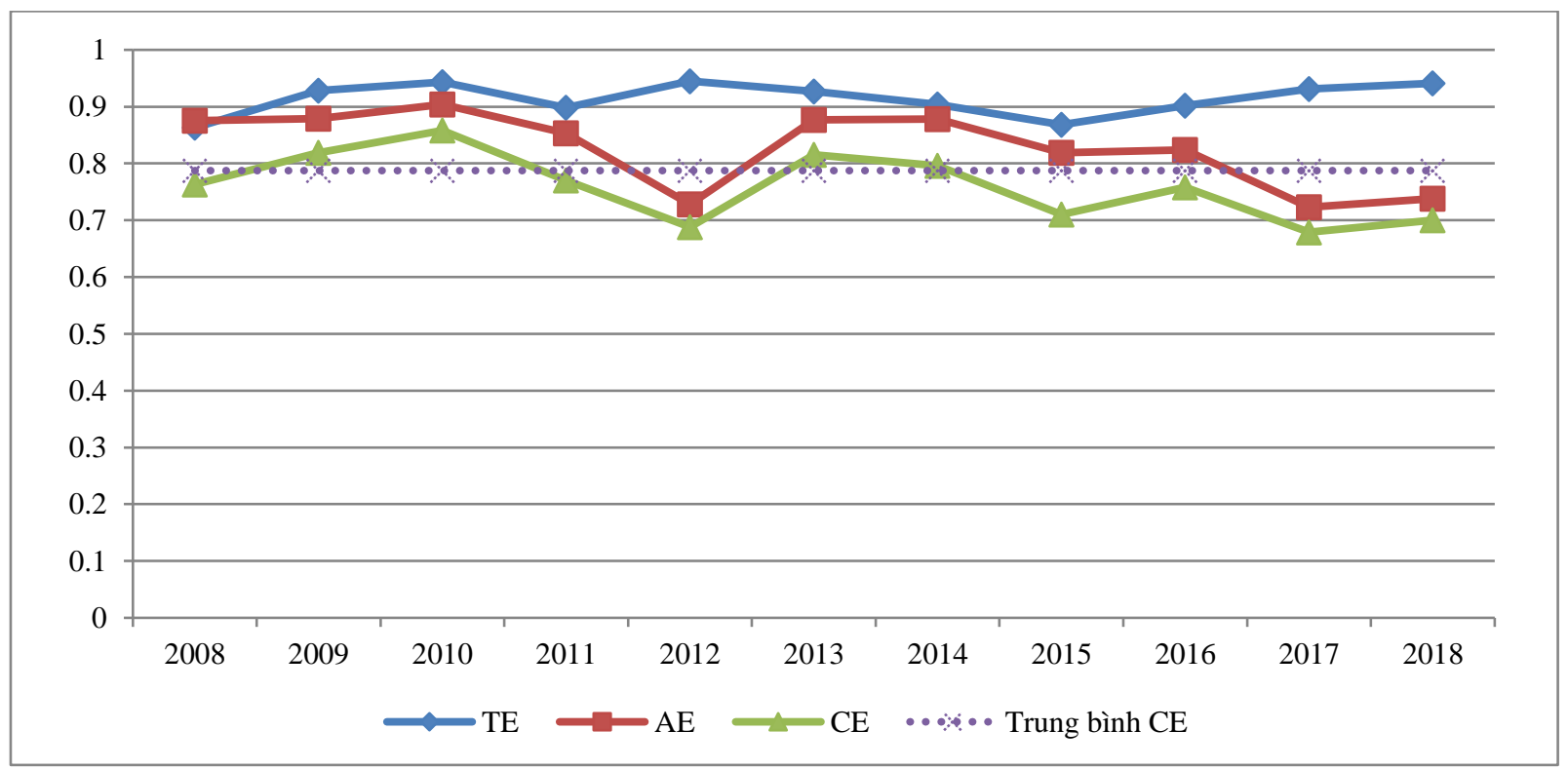

Hình 1: Hiệu quả kỹ thuật, hiệu quả phân bổ và hiệu quả chi phí trung bình của các NHTM Việt Nam trong giai đoạn 2008-2018

Nguồn: Tính toán của nhóm tác giả

4.2 Mức độ đổi mới công nghệ tại các ngân hàng thương mại Việt Nam trong giai đoạn 2008-2018

Kết quả phân rã chỉ số Malmquist trong DEA cho thấy chỉ số đổi mới công nghệ (TECH) trung bình của các NHTM Việt Nam trong giai đoạn 2008-2018 ở mức 0,976<1, giảm 2,4\% toàn giai đoạn (Bảng 5 ). Điều này phản ánh mức độ đổi mới công nghệ của các NHTM Việt Nam vẫn chưa cao, tiêu biểu như các ngân hàng $\mathrm{ABB}, \mathrm{ACB}, \mathrm{BIDV}, \mathrm{CTG}, \mathrm{EIB}, \mathrm{NAB}, \mathrm{OCB}, \mathrm{SCB}, \mathrm{SEA}, \mathrm{SGB}, \mathrm{TCB}, \mathrm{VAB}, \mathrm{VCB}, \mathrm{VIB}$ đều có có hệ số $\mathrm{TECH}<1$, trong đó phải kể đến các NHTM cổ phần có vốn Nhà nước có quy mô lớn nhất tại Việt Nam 
hiện nay như BIDV, CTG, VCB đều chưa chú trọng đầu tư vốn mạnh vào cải tiến công nghệ ngân hàng. Chỉ có một số ít ngân hàng như $\mathrm{HDB}, \mathrm{MBB}, \mathrm{MSB}, \mathrm{SHB}, \mathrm{TPB}, \mathrm{VPB}$ tập trung đẩy mạnh cải tiến công nghệ $(\mathrm{TECH}>1)$. Kết quả trên cho thấy, trong bối cảnh cách mạng công nghiệp 4.0 hiện nay, các NHTM Việt Nam cũng đã chú trọng đầu tư đổi mới công nghệ để nâng cao hiệu quả ngân hàng, song nhìn chung mức độ thay đổi tiến bộ công nghệ vẫn chưa cao, các NHTM Việt Nam vẫn còn chú trọng nhiều về những công nghệ sử dụng nhiều lao động.

Bảng 5: Chỉ số đổi mới công nghệ trung bình của các NHTM Việt Nam trong giai đoạn 2008-2018

\begin{tabular}{|c|c|c|c|c|c|c|c|c|c|c|c|}
\hline Ngân hàng & ABB & ACB & BIDV & CTG & EIB & HDB & MBB & MSB & NAB & OCB & PGB \\
\hline TECH & 0,958 & 0,979 & 0,965 & 0,925 & 0,972 & 1,013 & 1,002 & 1,033 & 0,957 & 0,943 & 0,974 \\
\hline Ngân hàng & SCB & SEA & SGB & SHB & STB & TCB & TPB & VAB & VCB & VIB & VPB \\
\hline TECH & 0,901 & 0,938 & 0,938 & 1,001 & 1,012 & 0,985 & 1,005 & 0,961 & 0,984 & 0,989 & 1,057 \\
\hline Trung bình & \multicolumn{10}{|c|}{$\mathbf{0 , 9 7 6}$} \\
\hline
\end{tabular}

Nguồn: Tính toán của nhóm tác giả

\subsection{Kết quả ước lượng mô hình hồi quy Tobit}

Kết quả ước lượng mô hình Tobit tại Bảng 6 cho thấy tốc độ đổi mới công nghệ có tác động cùng chiều đến hiệu quả chi phí của các NHTM Việt Nam tại mức ý nghĩa thống kê $1 \%$. Điều này có nghĩa là khi tốc độ đổi mới công nghệ tăng thì sẽ làm tăng hiệu quả chi phí của các NHTM Việt Nam và ngược lại. Hình 3 cho thấy, trong các năm 2010, 2013, 2016, 2018, khi tốc độ đổi mới công nghệ tăng lên tương ứng với các mức $1,064-1,008-1,171-1,102$ thì hiệu quả chi phí của các NHTM Việt Nam cũng tăng tương ứng với các mức $0,858-0,815-0,758-0,7$. Ngược lại, khi tốc độ đổi mới công nghệ giảm xuống trong các năm $2009,2011,2012,2014,2015,2017$ tương ứng với các mức $0,811-1,038-0,83-0,946-0,925-0,996$ thì hiệu quả chi phí của các NHTM Việt Nam cũng giảm tương ứng với các mức $0,819-0,77-0,688-$ $0,796-0,71-0,679$. Kết quả nghiên cứu chấp nhận giả thuyết $\mathrm{H} 1$ và tương đồng với kết quả nghiên cứu của Beccalli (2007), Ngugi \& Karina (2013), Yang \& Huang (2009). Phát hiện này hàm ý rằng vấn đề đổi mới công nghệ đóng vai trò quan trọng trong việc nâng cao hiệu quả của các NHTMVN.

Bên cạnh đó, kết quả mô hình Tobit cũng cho thấy các biến kiểm soát gồm khả năng sinh lời, sở hữu nhà nước, tăng trưởng kinh tế, lãi suất có tác động cùng chiều, trong khi đó nợ xấu và lạm phát tác động cùng chiều đến hiệu quả chi phí của các NHTM Việt Nam tại mức ý nghĩa thống kê $1 \%$ và $5 \%$. Kết quả này tương đồng với kết quả nghiên cứu của Sari \& Saraswati (2017), Hassan \&Sanchez (2007), Sufian \& Noor (2009), Havrylchyk (2006), Vu \& Nahm (2013), GarzaGarcía (2012).

Bảng 6: Kết quả ước lượng mô hình hồi quy Tobit thể hiện tác động của đổi mới công nghệ đến hiệu quả của các

NHTM Việt Nam trong giai đoạn 2008-2018

\begin{tabular}{|c|c|c|c|}
\hline Biến & Hệ số hồi quy & Sai số chuẩn & z \\
\hline TECH & $0,5309^{* * *}$ & 0,0744 & 7,14 \\
\hline ROA & $0,0745^{* * *}$ & 0,0265 & 2,81 \\
\hline NPL & $-0,0350^{* * *}$ & 0,0110 & $-3,17$ \\
\hline SIZE & 0,0117 & 0,0296 & 0,40 \\
\hline EA & 0,0047 & 0,0055 & 0,86 \\
\hline SO & $-0,1614^{* *}$ & 0,0660 & $-2,44$ \\
\hline FO & $-0,0079$ & 0,0594 & $-0,13$ \\
\hline GDP & $0,0498^{* *}$ & 0,0246 & 2,02 \\
\hline INF & $-0,0099^{* * *}$ & 0,0032 & $-3,13$ \\
\hline RATE & $0,0170^{* *}$ & 0,0065 & 2,60 \\
\hline C & $1,0318^{* * *}$ & 0,3249 & 3,18 \\
\hline
\end{tabular}

Ghi chú: *, **, *** Ý nghĩa thống kê lần lươt ở mức 10\%, 5\% và 1\%

Nguồn: Tính toán của nhóm tác giả 


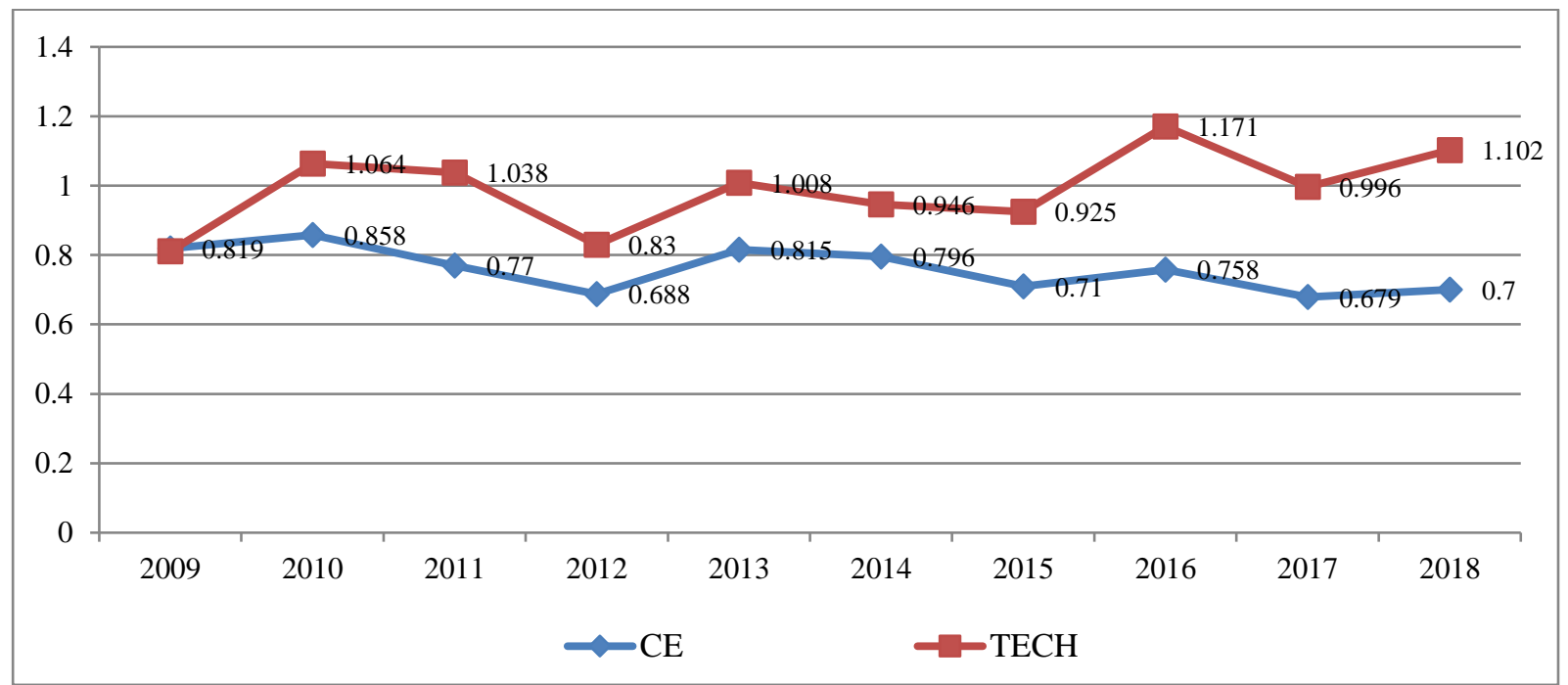

Hình 3: Đổi mới công nghệ và hiệu quả chi phí của các NHTM Việt Nam trong giai đoạn 2008-2018

Nguồn: Tính toán của nhóm tác giả

\section{KẾT LUẬN VÀ HÀM Ý}

Bài viết này nghiên cứu tác động của đổi mới công nghệ đến hiệu quả của 22 NHTM Việt Nam trong giai đoạn 2008-2018. Đầu tiên, nghiên cứu đo lường hiệu quả và chỉ số đổi mới công nghệ của các NHTM Việt Nam trong giai đoạn 2008-2018 theo cách tiếp cận DEA. Sau đó, nghiên cứu sử dụng mô hình hồi quy Tobit để xem xét tác động của đổi mới công nghệ đến hiệu quả của các NHTM Việt Nam. Kết quả nghiên cứu cho thấy: Tốc độ đổi mới công nghệ trung bình của các NHTM Việt Nam ở mức mức $0,976<1$, giảm $2,4 \%$ toàn giai đoạn. Trong khi đó, hiệu quả chi phí trung bình của các NHTM Việt Nam ở mức 78,74\%, cho thấy các NHTM Việt Nam chưa sử dụng tối đa các nguồn lực đầu vào. Kết quả mô hình Tobit cho thấy chỉ số đổi mới công nghệ có tác động cùng chiều đến hiệu quả chi phí của các NHTM Việt Nam. Ngoài ra, nghiên cứu này cũng chỉ ra rằng khả năng sinh lời, tăng trưởng kinh tế, lãi suất có tác động cùng chiều, trong khi đó sở hữu nhà nước, nợ xấu và lạm phát tác động ngược chiều đến hiệu quả chi phí của các NHTM Việt Nam. Kết quả nghiên cứu mang lại đóng góp mới về phương pháp tiếp cận đo lường chỉ số đổi mới công nghệ và bổ sung vào khoảng trống nghiên cứu thông qua cung cấp bằng chứng thực nghiệm về tác động của đổi mới công nghệ đến hiệu quả của các NHTM Việt Nam.

Từ kết quả nghiên cứu nêu trên, trong thời gian tới, để nâng cao hiệu quả ngân hàng, các NHTM Việt Nam cần tiếp tục tăng cường đổi mới công nghệ trên cơ sở tiếp tục đầu tư, phát triển các sản phẩm công nghệ số; đẩy mạnh công tác triển khai các ứng dụng công nghệ vào hoạt động ngân hàng; tăng cường đào tạo nguồn nhân lực công nghệ cao; tăng cường hợp tác bền vững với các công ty Fintech nhằm đa dạng thêm các sản phẩm, dịch vụ cung ứng cho khách hàng, qua đó hạn chế rủi ro do đầu tư chi phí quá lớn vào việc đổi mới công nghệ ngân hàng. Bên cạnh đó, các NHTM tiểp tục nâng cao năng lực tài chính thông qua việc giảm dần tỷ lệ sở hữu nhà nước, nâng cao khả năng sinh lời, tiếp tục xử lý triệt để nợ xấu. Về phía Chính phủ và các cơ quan chức năng, cần tiếp tục thực hiện chính sách thúc đẩy tăng trưởng kinh tế, kiềm chế lạm phát, điều hành lãi suất theo hướng linh hoạt, ổn định hệ thống ngân hàng, rà soát và ban hành các chính sách, tạo môi trường pháp lý thuận lợi cho hoạt động của các NHTM Việt Nam.

\section{TÀI LIÊU THAM KHẢO}

[1] Abaenewe, Z. C., Ogbulu, O. M., \& Ndugbu, M. O. (2013). Electronic banking and bank performance in Nigeria. West African journal of industrial and academic research, 6(1), 171-187.

[2] Adjei-Frimpong, K., Gan, C., \& Hu, B. (2014). Cost efficiency of Ghana's banking industry: A panel data analysis. The International Journal of Business and Finance Research, 8(2), 69-86.

[3] Aigner, D.J.; Lovell, C.A.K.; Schmidt, P. (1977) Formulation and estimation of stochastic frontier production functions. Journal of Econometrics, 6:21-37. 
[4] Ariff, M., \& Can, L. (2008). Cost and Profit Efficiency of Chinese Banks: A Non-Parametric Analysis. China Economic Review, 19, 2, 260-273.

[5] Banker R.D., A. Charnes and W.W. Cooper, (1984), "Some Models For Estimating Technical and Scale Inefficiencies In Data Envelopment Analysis", Management Science.

[6] Batir, T. E., Volkman, D. A., \& Gungor, B. (2017). Determinants of bank efficiency in Turkey: Participation banks versus conventional banks. Borsa Istanbul Review, 17(2), 86-96.

[7] BCG (2009). Boston Consulting Group - BCG Innovation 2009 Report.

[8] Beccalli E (2007) Does IT Investment Improve Bank Performance? Evidence from Europe. J Banking Finance 31: 2205-2230.

[9] Berger, A. \& Humphrey, D. (1992). Measurement and Efficiency Issues in Commercial Banking. National Bureau of Economic Research, 245-300.

[10] Campanella, F., Della Peruta, M. R., \& Del Giudice, M. (2017). The effects of technological innovation on the banking sector. Journal of the Knowledge Economy, 8(1), 356-368.

[11] Chaarani \& Abiad (2018). The impact of technological innovation on bank performance. Journal of Internet Banking and Commerce. http://www.icommercecentral.com/open-access/the-impact-of-technologicalinnovation-on-bank-performance.php?aid=87293.

[12] Chandra, P., Cooper, W. W., Li, S., \& Rahman, A. (1998). Using DEA to evaluate 29 Canadian textile companies - considering returns to scale. International Journal of Production Economics, 54(2), 129-141.

[13] Charnes, A., Cooper, W. \& Rhodes, E. (1978). Measuring the efficiency of decision-making units. European Journal of Operational Research, 2, 429-444.

[14] Cruz-Cázares, C., Bayona-Sáez, C., \& García-Marco, T. (2013). You can’t manage right what you can’t measure well: Technological innovation efficiency. Research policy, 42(6-7), 1239-1250.

[15] DeYoung R (2001) The Financial Performance of Pure Play Internet Banks. Econ Perspectives 25: 60-75.

[16] Färe, R, S Grosskopf and CAK Lovell (1994). Production Frontiers, Cambridge: University Press Cambridge.

[17] Farrell, M. J. (1957). The Measurement of Productive Efficiency. Journal of the Royal Statistical Society, 120 (3) 253-81.

[18] Garza-García, J. G. (2012). Determinants of bank efficiency in Mexico: a two-stage analysis. Applied Economics Letters, 19(17), 1679-1682.

[19] Gujarati, D. (2003). Basic econometrics. McGraw Hill, Boston, USA.

[20] Hassan, M. K., \& Sanchez, B. (2007). Efficiency determinants and dynamic efficiency changes in Latin American banking industries. Available at SSRN 3263102.

[21] Havrylchyk, O. (2006). Efficiency of the Polish Banking Industry: Foreign Versus Domestic Banks. Journal of Banking and Finance, 30, 7, 1975-1996.

[22] Hoàng,T. H. \& Huân, N. H. (2016). Phân tích các yếu tố tác động đến hiệu quả hoạt động của hệ thống ngân hàng thương mại Việt Nam trong thời kỳ hội nhập tài chính quốc tế. Tạp chí Phát Triển KH \& CN, Tập 19, Số Q1 - 2016, 88-101.

[23] Hobe 1, Alas R (2016). A Financial Innovation Management Model For Banks. J Manage p: 138-155.

[24] Kablan, S. (2009). Banking Efficiency and Financial Development in Sub-Saharan Africa. African Finance Journal, 11(2), 28-50.

[25] Khalil, M (2000), Management of technology, McGraw-Hill, Burr Ridge, IL.

[26] Kumar, M., \& Vincent, C. (2011). Benchmarking Indian banks using DEA in post-reform period: a progressive time-weighted mean approach. The Service Industries Journal, 31(14), 2455-2485.

[27] Kumar, S., \& Arora, N. (2011). Assessing technical efficiency of sugar industry in Uttar Pradesh: an application of data envelopment analysis. Indian Economic Review, 323-353.

[28] Kumar, S., \& Gulati, R. (2009). Technical efficiency and its determinants in the Indian domestic banking industry: an application of DEA and Tobit analysis. American Journal of Finance and Accounting, 1(3), 256296.

[29] Langley et al., (2011). Credit derivatives and bank credit supply ${ }^{\text {ee }}$ Staff Report No 276-2007. Federal Reserve Bank of New York.

[30] Liu, F. C., \& Pan, X. F. (2007). Evaluate of science and technology innovation efficiency based on malmquist index approach [J]. Studies in Science of Science, 5. 
[31] Mercan, M., Arnold R., Reha Y., \& Ahmet, B. E. (2003). The Effect of Scale and Mode of Ownership on the Financial Performance of the Turkish Banking Sector: Results of a DEA-Based Analysis. Socio-Economic Planning Sciences, 37, 185-202.

[32] Ngugi, K. \& Karina, B. (2013), Effect of Innovation Strategy on performance of Commercial Banks in Kenya. International Journal of Social Sciences and Entrepreneurship,Vol.1, Issue 3, 2013, 1 (3), 158-170.

[33] Oke and Goffin (2011). Technology adoption and consumer payments: evidence from survey data. Review of Network Economics, 2(2).

[34] Oligopolistic Market: Evidence from Singapore. Journal of Multinational Financial Management 8, $155-168$.

[35] Ratten, V. (2008). Technological innovations in the m-commerce industry: A conceptual model of WAP banking intentions. The Journal of High Technology Management Research, 18(2), 111-117.

[36] Rezitis, N. A. (2007). Efficiency and Productivity Effects of Bank Mergers: Evidence from the Greek Banking Industry. Economic Modelling, 25, 236-254.

[37] Rishi,M., \& Saxena, S. C. (2004). Technological innovations in the Indian banking industry: the late bloomer. Accounting, Business \& Financial History, 14(3), 339-353.

[38] Rogers, E. (1962). Diffusion of innovations. New York: The Free Press.

[39] Romdhane, S. B. (2013) Impact of Information Technology on the Performance of Tunisian Banks: A Stochastic Frontier Analysis with Panel Data. Asian Academy Manage J Accounting Financ 9: 95-125.

[40] Said, J., Hasnan, S., Ismail, F., Majid, M. S. A., \& Rahim, R. A. (2013). Efficiency of Islamic and conventional banks in Malaysia. Journal of Financial Reporting and Accounting.

[41] Sáng, N. M. (2013). Phân tích nhân tố tác động đến hiệu quả sử dụng nguồn lực của các ngân hàng thương mại trên địa bàn TPHCM. Tạp chí Phát triển \& Hội nhập, Số 11 (21) - Tháng 07-08/2013, 10-15.

[42] Sari, P. Z., \& Saraswati, E. (2017). The Determinant of Banking Efficiency in Indonesia (DEA Approach). Journal of Accounting and Business Education, 1(2), 208-229.

[43] Sealey, C. \& Lindley, J. (1977). Inputs, Outputs and Theory of Production Cost at Depository Financial Institutions. Journal of Finance 32 (4) 1251-1266.

[44] Sufian, F., \& Noor, M. A. N. M. (2009). The determinants of Islamic banks' efficiency changes. International Journal of Islamic and Middle Eastern Finance and Management.

[45] Thương, N. T. T. (2017). Hiệu quả hoạt động của các ngân hàng thương mại trên địa bàn tỉnh Thái Nguyên. Tạp chí Khoa học Trường đại học Cần Thơ. Tập 50, Phần D (2017): 52-62.

[46] Tobin, J. (1958), "Estimation of Relationships for Limited Dependent Variables", Econometrica, 26 (1), $24-36$.

[47] Trúc, L. T. \& Danh, V. T. (2012). Phân tích các nhân tố ảnh hưởng đến hiệu quả hoạt động kinh doanh của hệ thống ngân hàng thương mại cổ phần việt nam giai đoạn 2006-2009. Tạp chí Khoa học 2012:21a 148-157.

[48] Vu, H., \& Nahm, D. (2013). The determinants of profit efficiency of banks in Vietnam. Journal of the Asia Pacific Economy, 18, 615-631.

[49] Williams, J., \& Nguyen, N. (2005). Financial Liberalisation, Crisis, and Restructuring: A Comparative Study of Bank Performance and Bank Governance in South East Asia. Journal of Banking and Finance, 29, 8-9, 21192154.

[50] Wozniewska, G. (2008). Methods of Measuring the Efficiency of Commercial Banks: An Example of Polish Banks. Ekonomika. ISSN 1392-1258.

[51] Wuqin, S., \& Shunlin, Z. (2008). The Efficiency of Finance Development on Improving Technological Innovation - Based on the Malmquist Index Analysis [J]. Statistical Research, 3.

[52] Yang, Y.L., Huang, C. J. (2009). Estimating the malmquist productivity index in the Taiwanese banking industry: A production and cost approach. Taiwan Economic Review 37(4), 353-378.

[53] Yuan, P., Chen, Q., \& Hu, R. (2007). Dynamic Change of Region Innovation Performance in China: An Analysis of Malmquist Index [J]. Science of Science and Management of S. \& T, 1.

[54] Yun, L. (2011). The efficiency study of regional technological innovation: Based on the provinces level. Energy Procedia, 5, 1579-1583.

Ngày nhận bài: 25/04/2021

Ngày chấp nhận đăng: 23/07/2021 